\title{
Effets des pratiques agro-écologiques sur les caractéristiques chimiques majeures et le stock de carbone du sol à l'Est du Burkina Faso
}

Moussa Gnissien, Kalifa Coulibaly, Mamadou Traore, Mipro Hien, Bertrand Mathieu \& Hassan Bismarck Nacro

Moussa Gnissien : Laboratoire d’Etude et de Recherche sur la Fertilité du Sol (LERF), Université Nazi BONI, Bobo-Dioulasso, BP 1091 Burkina Faso. Auteur correspondant : Tel : 00226717956

16 Email : moussa.gnissien@yahoo.fr

Kalifa Coulibaly : Laboratoire d’Etude et de Recherche sur la Fertilité du Sol (LERF), Université Nazi BONI, Bobo-Dioulasso, BP 1091 Burkina Faso

Mamadou Traore : Laboratoire d'Etude et de Recherche sur la Fertilité du Sol (LERF), Université Nazi BONI, Bobo-Dioulasso, BP 1091 Burkina Faso

Mipro Hien : Laboratoire des Systèmes Naturels, Agro-systèmes et de l'Ingénierie de l'Environnement (Sy.N.A.I.E), Université Nazi BONI, Bobo-Dioulasso, BP 1091 Burkina Faso

Bertrand Mathieu : Agronomes et Vétérinaires Sans Frontières

Hassan Bismarck Nacro : Laboratoire d’Etude et de Recherche sur la Fertilité du Sol (LERF), Université Nazi BONI, Bobo-Dioulasso, BP 1091 Burkina Faso

DOI: $10.25518 / 2295-8010.1862$

\section{Résumé :}

Les pays sahéliens sont confrontés au problème récurrent de la baisse de fertilité des sols. En réponse à cette baisse de fertilité des sols, diverses stratégies sont mises en œuvre par les producteurs en vue d'améliorer la fertilité des sols. L'étude a été conduite en milieu paysan, dans les villages de Bilanga yanga, Tiguili et Kolomi à l'Est du Burkina Faso. Elle a eu pour objectif d'évaluer les impacts de pratiques agro-écologiques sur les caractéristiques chimiques, le stock de carbone du sol et les rendements de sorgho. Les entretiens avec les producteurs ont permis de retenir les pratiques les plus répandues. Ce sont : Cordons Pierreux (CP) + Enfouissement de la paille de céréales (Enf) + Fumure Organique (FO) (5 parcelles), CP + FO (5 parcelles), CP + $\mathrm{Zaï} \mathrm{+} \mathrm{FO} \mathrm{(5} \mathrm{parcelles),} \mathrm{Enf} \mathrm{+} \mathrm{FO} \mathrm{(4} \mathrm{parcelles),} \mathrm{FO} \mathrm{(5} \mathrm{parcelles)} \mathrm{et} \mathrm{Témoin} \mathrm{(5} \mathrm{parcelles).}$ Globalement, les pratiques intégrant les cordons pierreux ont amélioré significativement ( $\mathrm{p}<$ 0,05 ) les caractéristiques chimiques et le stock de carbone du sol. Les résultats ont montré une augmentation du stock de carbone de $20 \%$ à $46 \%$ pour les pratiques intégrant les cordons pierreux. Les pratiques agro-écologiques comportant les cordons pierreux seraient-elles les plus performantes dans l'amélioration de la fertilité des sols à l’Est du Burkina Faso ?

Mots-clés : cordons pierreux, zaï, fumure organique, enfouissement de résidus, fertilité du sol

\section{Abstract :}


Effets des pratiques agro-écologiques sur les caractéristiques chimiques maje...

The Sahelian countries are faced with the recurrent problem of declining soil fertility. In response to this decline in soil fertility, various strategies are implemented by producers to improve soil fertility.The study took place in farmer's fields in the villages of Bilanga yanga, Tiguili and Kolokomi (in eastern Burkina Faso). The objective was to assess the impacts of agroecological practices on chemical characteristics, soil carbon stock and sorghum yields. The interviews with the producers made it possible to select the most widespread practices. These practices are: Stony lines (CP) + Burying Cereal Straw (Enf) + Organic Fumure (FO) (5 repetitions), $\mathrm{CP}+\mathrm{FO}$ (5 repetitions), $\mathrm{CP}+$ Zaï (5 repetitions), Enf + FO (4 repetitions), FO (5 repetitions) and Control (5 repetitions). For the evaluation of sorghum yields, four (4) practices were selected: CP + Enf + FO, CP + Zaï, Enf + FO and Control. Overall, practices incorporating stone lines recorded the highest grades $(\mathrm{p}<0,05)$ for chemical characteristics and soil carbon stock. The results showed an increase in carbon stock showed from $20 \%$ to $46 \%$ of practices incorporating stony lines. Are agro-ecological practices with stony lines the most effective in improving soil fertility in eastern Burkina Faso?

Keywords : stony lines, zaï, organic manure, burying of residues, soil fertility, carbon stock.

\section{Introduction}

L'agriculture burkinabé est confrontée à d'importantes contraintes limitant sa production (16). Parmi ces contraintes figurent les conditions pédoclimatiques défavorables (20) et les facteurs anthropiques basés sur la pratique d'une agriculture extensive avec un faible niveau de restitutions organiques (3). Ces contraintes ont accéléré la dégradation des terres entrainant une baisse de la fertilité des sols $(7 ; 13 ; 14)$. Face à cette baisse de la fertilité des sols, les producteurs ont expérimenté des pratiques mettant l'accent sur les processus naturels de gestion des agrosystèmes, dites pratiques agro-écologiques. Les pratiques agro-écologiques désignent des techniques utilisant les ressources et processus naturels pour améliorer la fertilité des sols et assurer la protection des cultures contre les bio agresseurs. Ainsi, l'agro-écologie a à son actif une gamme variée de composantes basées sur les pratiques naturelles endogènes dont la mise en œuvre est fonction des réalités locales de chaque région (22).

Dans différentes localités du Burkina Faso, les cordons pierreux, le zaï, l'enfouissement des résidus de récolte / paillage et l'apport de la fumure organique en tant que techniques CES/DRS ont été utilisés individuellement ou en combinaison comme techniques de restauration et/ou de maintien des propriétés physiques et chimiques du sol $(17 ; 24 ; 29)$. Aussi, des investigations sur les pratiques de l'agriculture de conservation, qui comprennent la réduction du travail du sol à la couverture permanente du sol et à l'association et / ou la rotation des cultures, rapportent une réduction du ruissellement (19) et l'augmentation du stock de carbone du sol $(6 ; 11 ; 21)$.

Ces pratiques concourent au même résultat que la mise en œuvre des techniques CES/DRS précédemment mentionnées. La plupart des travaux portant sur l'effet des techniques CES / DRS sur la fertilité des sols a été effectuée dans les parties Nord, Centre-Nord et Centre du pays, pourtant ces techniques sont aussi mises en œuvre par les producteurs dans les parties comme l'Est. Généralement l'effet des techniques CES / DRS a été évalué sur le court-moyen terme. En plus, des travaux réalisés, la contribution de ces techniques au stockage du carbone dans le sol a été peu abordée. Cet article présente les effets à moyen-long termes des pratiques agro-écologiques sur les caractéristiques chimiques majeures et le stock de carbone des sols à l'Est du Burkina Faso. 


\section{Matériel et méthodes}

\section{Milieu d'étude}

Létude a été conduite dans la région Est du Burkina Faso, dans la commune rurale de Bilanga, située à l'extrême sud de la province de la Gnagna, entre $12^{\circ} 32^{\prime} 52^{\prime \prime}$ de latitude Nord et $0^{\circ} 2^{\prime} 10^{\prime \prime}$ de longitude Ouest, avec une altitude de $271 \mathrm{~m}$. Les données ont été collectées dans les villages de Bilanga Yanga, Tiguili et Kolokomi. Le milieu d'étude appartient au secteur nord soudanien (10), avec un climat de type soudano-sahélien et des précipitations annuelles moyennes de l'ordre de $700 \mathrm{~mm}$. Plusieurs types de sols sont rencontrés dans la province de la Gnagna, mais les lixisols (27) sont les plus dominants (5). Ce sont des sols carencés en phosphore, avec une faible teneur en matière organique (moins de $1 \%$ ), la somme des bases échangeables et la Capacité d'échéance cationique sont basses à moyennes. Ces sols sont rencontrés sur les plateaux, les pentes de glacis (hauts, bas et moyennes) avec des textures variables : sablo-limoneuse, limono-sableuse, argilosableuse (5).

\section{Collecte des données}

La méthode active d'approches et de recherche participative (MARP) a été utilisée pour la collecte des données et cela à travers le déroulement de certains outils tels que le transect pour la collecte des échantillons de sol, le choix des champs pour le suivi agronomique et la description de l'environnement physique, les interviews semi-structurées en groupes cible et individuelles, les entretiens avec les services techniques et autres personnes de ressources pour la collecte et/ou pour recouper les informations socio-économiques. La seconde phase de la collecte des données a consisté au suivi des parcelles dans les champs des producteurs retenus à l'issue des différentes phases d'entretiens.

\section{Caractérisation des pratiques agro-écologiques et choix des traitements}

Une pré-enquête effectuée en mai 2017 sous forme de focus group avec 97 producteurs a permis d'identifier en plus de la pratique Témoin, cinq (05) types de pratiques agro-écologiques combinées ou pas. Des interviews individuelles auprès de 58 producteurs sélectionnés de façon aléatoire, a permis de retenir 29 parcelles où sont mises en œuvre différentes pratiques agro-écologiques (Tableau 1).

Cette enquête a également permis de relever la durée de mise en œuvre des pratiques agroécologiques par les producteurs. La réalisation des cordons pierreux et l'enfouissement de la paille de céréales sont mises en œuvre depuis $12 \pm 8$ ans en moyenne, la fumure organique est apportée sur les parcelles depuis $11 \pm 7$ ans et le zaï est pratiqué depuis $4 \pm 1$ ans. Les cordons pierreux sont placés suivant les courbes de niveau et sont espacés de $34 \pm 5 \mathrm{~m}$, la paille de céréales est enfouie annuellement par le labour à une quantité de $1080 \pm 372 \mathrm{~kg} /$ ha. La fumure organique est appliquée annuellement par épandage avant le labour à une dose de $2475 \pm 447 \mathrm{~kg} / \mathrm{ha}$. Les trous de zaï sont creusés manuellement en fin de saison sèche, et la fumure organique est déposée au fond des trous sur une année. La fumure organique associée aux différentes pratiques agroécologiques est généralement sous la forme de compost obtenu par décomposition de la biomasse et de déjections animales. 
Effets des pratiques agro-écologiques sur les caractéristiques chimiques maje...

Tableau 1: Pratiques agro-écologiques mises en œuvre par les producteurs de la zone d'étude

\begin{tabular}{|l|l|l|}
\hline Pratiques agro-écologiques & Abréviation & $\begin{array}{l}\text { Nombre total de } \\
\text { parcelles (29) }\end{array}$ \\
\hline $\begin{array}{l}\text { Cordons Pierreux + Enfouissement de la paille de céréales + } \\
\text { Fumure Organique }\end{array}$ & $\begin{array}{l}\text { CP + Enf + } \\
\text { FO }\end{array}$ & 5 \\
\hline Cordons Pierreux + Fumure Organique & CP + FO & 5 \\
\hline Cordons Pierreux + Zaï + FO & $\begin{array}{l}\text { CP + Zaï + } \\
\text { FO }\end{array}$ & 5 \\
\hline Enfouissement de la paille de céréales + Fumure Organique & Enf + FO & 4 \\
\hline Fumure Organique & FO & 5 \\
\hline Témoin & Témoin & 5 \\
\hline
\end{tabular}

\section{Collecte des échantillons de sol}

La plupart des parcelles étaient situées sur des moyennes et bas de pentes de glacis. Les précédents culturaux étaient constitués principalement de sorgho et de mil qui sont les spéculations les plus produites dans la zone. L'engrais NPK et l'urée sont les fertilisants minéraux utilisés. Il faut cependant signaler que leur utilisation par les producteurs demeure marginale en raison de leur coût jugé élevé par ces derniers.

Les prélèvements de sol ont été effectués avant les semis de la campagne 2017-2018. Ainsi, suivant une diagonale, trois échantillons de sol ont été prélevés respectivement sur les profondeurs 0-10 $\mathrm{cm}$ et 10-20 cm du sol pour la détermination des caractéristiques chimiques majeures du sol. Concernant le stock de carbone, les prélèvements ont concerné uniquement la profondeur 0-10 cm pour l'évaluation du stock de carbone. Dans le cas des parcelles avec cordons, les prélèvements ont été effectués entre les cordons pierreux à au moins $2 \mathrm{~m}$ des cordons. Pour les parcelles avec zaï, les prélèvements ont été effectués dans les trous et entre les trous de zaï et mélanger pour obtenir des échantillons composites.

\section{Analyses chimiques du sol}

Les échantillons de sol prélevés ont été séchés à l'air libre et à l'ombre avant d'être tamisés à 2 $\mathrm{mm}$ pour obtenir la fraction fine. Les analyses ont porté sur les $\mathrm{pH}$ eau et $\mathrm{pH} \mathrm{KCl}(1)$, le carbone total (26), l'azote total (12), le phosphore total et le potassium total (18), le phosphore assimilable avec la méthode de Bray I (4). Quant au potassium disponible, il a été extrait selon la méthode de l'acétate d’ammonium.

Ces analyses ont été réalisées par le Laboratoire Sols-eaux-plantes de l’Institut National de Recherches Agricoles et Environnementales à Farako-Bâ (Burkina Faso). 


\section{Détermination du stock de carbone}

La détermination du stock de carbone du sol a été faite selon la méthode de (2). Pour cela, des carottes de sol prélevées à l'aide d'un cylindre de volume connu ont été séchées à l'étuve à $105^{\circ} \mathrm{C}$ pendant 24 heures, suivi de la détermination de la densité apparente selon la formule (I)

(I) $\rho=\frac{M}{V}$

avec $\rho=$ densité apparente (en g. $\mathrm{cm}^{-3}$ ),

M= masse de l'échantillon de sol séché à l'étuve (en g) et ,

V son volume en $\mathrm{cm}^{3}$.

Le calcul du stock de carbone se fait en multipliant la concentration de carbone (\%) de la fraction fine du sol (fraction de diamètre $<2 \mathrm{~mm}$ ) par la densité apparente $(\rho)$, la profondeur de sol prélevé (D) et la proportion de la fraction fine du sol ( comme suit :

$$
(\text { II }) \operatorname{SOC}(g C c m-2)=\frac{C}{100} \times \rho \times D \times(1-\text { frag })
$$

Le stock de carbone sur la profondeur 0-10 $\mathrm{cm}$ a été exprimé en $\mathrm{t}$. ha ${ }^{-1}$ en convertissant les grammes ( $\mathrm{g}$ ) en tonnes (t) et les $\mathrm{cm}^{-2}$ en ha ${ }^{-1}$; ce qui revient à multiplier l'équation (1) par 100.

La fraction grossière (frag) a été obtenue par tamisage à $2 \mathrm{~mm}$ des échantillons de sol utilisés pour la détermination de la densité apparente. Les refus de taille supérieure ou égale à $2 \mathrm{~mm}$ qui ont constitué la fraction grossière ont ainsi été pesés. Le poids de la fraction fine a été calculé à partir de celui de la fraction grossière par la formule fraction fine $=(1$ - frag $)$.

La charge en éléments grossiers (EG) a été calculée en faisant le rapport du poids de la fraction grossière par celui de la carotte de sol séché à l'étuve à $105^{\circ} \mathrm{C}$.

EG $(\%)=$ frag $/$ Poids carotte de sol $* 100$

\section{Analyses statistiques}

Les données de sol et des rendements ont été soumises à une Analyse de Variance (ANOVA) avec le logiciel R, version 3.4.2. Avant de procéder à l'ANOVA, la normalité des données a été vérifiée par le test de Shapiro-Wilk. 


\section{Résultats}

\section{Effets des pratiques agro-écologiques sur les caractéristiques chimiques majeures du sol}

Les résultats des analyses chimiques des échantillons de sols des différentes pratiques agroécologiques sont consignés dans le Tableau 2. Pour la profondeur de sol 0-10 cm, le pH eau a varié de 6,39 (FO) à 7,07 ( $\mathrm{CP}+\mathrm{Zaï})$; le $\mathrm{pH}_{\mathrm{KCl}}$ a varié de 5,28 (Témoin) à 6,28 ( $\mathrm{CP}+\mathrm{Zaï).} \mathrm{Au} \mathrm{niveau}$ des éléments totaux, les teneurs sont comprises entre $0,49 \%$ et 1,23\% pour le carbone ; 0,04\% et $0,12 \%$ pour l'azote et 116 et $269 \mathrm{mg} / \mathrm{kg}$ de sol pour le phosphore total La pratique CP + FO a enregistré les plus fortes teneurs en carbone $(1,23 \%)$, azote $(0,12 \%)$ et phosphore total (269 mg / $\mathrm{kg}$ de sol).

Le Témoin a enregistré les plus faibles teneurs en carbone $(0,49 \%)$, azote $(0,04 \%)$ et phosphore total (116 mg / $\mathrm{kg}$ de sol). Les teneurs en phosphore assimilable sont comprises entre 2,7 mg (FO) et $14,7 \mathrm{mg} / \mathrm{kg}$ de sol $(\mathrm{CP}+\mathrm{FO})$. Pour le potassium disponible, les teneurs sont comprises entre 89 $\mathrm{mg} / \mathrm{kg}$ de sol (Témoin) et $414 \mathrm{mg} / \mathrm{kg}$ de sol (CP + Zaï). Les valeurs des caractéristiques chimiques sur la profondeur 10-20 cm ont évolué dans le même sens que sur celle de 0-10 cm. L'analyse de variance (ANOVA) a montré une différence significative (au seuil de $5 \%$ ) entre les pratiques pour les différentes caractéristiques chimiques du sol quelle que soit la profondeur. 
Tropicultura Tropicultura 2295-8010 Volume 39 (2021) Numéro 3, 1862

Tableau 2: Effets des pratiques agro-écologiques sur les caractéristiques chimiques majeures du sol 
Effets des pratiques agro-écologiques sur les caractéristiques chimiques maje...

\begin{tabular}{|c|c|c|c|c|c|c|c|c|}
\hline $\begin{array}{l}\text { Profondeur } \\
(\mathrm{cm})\end{array}$ & $\begin{array}{l}\text { Pratiques } \\
\text { AE }\end{array}$ & pHeau & pH & $\begin{array}{l}\text { C total } \\
(\%)\end{array}$ & $\begin{array}{l}\mathrm{N} \text { total } \\
(\%)\end{array}$ & $\begin{array}{l}\text { P total } \\
(\mathrm{mg} / \mathrm{kg} \\
\text { sol) }\end{array}$ & \begin{tabular}{|l|} 
P ass \\
$(\mathrm{mg} / \mathrm{kg}$ \\
sol)
\end{tabular} & $\begin{array}{l}\text { K dispo } \\
(\mathrm{mg} / \mathrm{kg} \\
\text { sol) }\end{array}$ \\
\hline & $\begin{array}{l}\mathrm{CP}+\mathrm{Enf}+ \\
\mathrm{FO}\end{array}$ & $\begin{array}{l}6,82 \pm \\
0,15^{\mathrm{ab}}\end{array}$ & $\begin{array}{l}5,71 \pm \\
0,23 \text { ab }\end{array}$ & $\begin{array}{l}0,87 \pm \\
0,14 \text { ac }\end{array}$ & $\begin{array}{l}0,07 \pm 0,01 \\
a b\end{array}$ & $\begin{array}{l}135,07 \pm \\
9,40^{\text {a }}\end{array}$ & $\begin{array}{l}3,78 \pm \\
0,75^{\mathrm{a}}\end{array}$ & $\begin{array}{l}140,58 \pm \\
25,52^{\text {a }}\end{array}$ \\
\hline & $\mathrm{CP}+\mathrm{FO}$ & $\begin{array}{l}6,94 \pm \\
0,07 \mathrm{ab}\end{array}$ & $\begin{array}{l}6,03 \pm \\
0,11^{\mathrm{ab}}\end{array}$ & $\begin{array}{l}1,23 \pm \\
0,07^{\mathrm{c}}\end{array}$ & $\begin{array}{l}0,12 \pm 0,01 \\
\mathrm{C}\end{array}$ & $\begin{array}{l}269,05 \pm \\
14,67^{b}\end{array}$ & $\begin{array}{l}14,65 \pm \\
2,02 \mathrm{~b}\end{array}$ & $\begin{array}{l}245,70 \pm \\
49,04 \text { ab }\end{array}$ \\
\hline & $\mathrm{CP}+\mathrm{Zaï}$ & $\begin{array}{l}7,07 \pm \\
0,26^{b}\end{array}$ & $\begin{array}{l}6,28 \pm \\
0,26^{\mathrm{b}}\end{array}$ & $\begin{array}{l}1,00 \pm \\
0,10 \mathrm{bc}\end{array}$ & $\begin{array}{l}0,08 \pm 0,01 \\
b\end{array}$ & $\begin{array}{l}169,94 \pm \\
34,27^{a}\end{array}$ & $\begin{array}{l}6,27 \pm \\
1,69 \mathrm{ab}\end{array}$ & $\begin{array}{l}413,77 \pm \\
91,57^{\mathrm{b}}\end{array}$ \\
\hline & Enf + FO & $\begin{array}{l}6,93 \\
\pm 0,20 \text { ab }\end{array}$ & $\begin{array}{l}6,03 \pm \\
0,30 \text { ab }\end{array}$ & $\begin{array}{l}0,51 \pm \\
0,07^{\mathrm{a}}\end{array}$ & $\begin{array}{l}0,05 \pm 0,01 \\
a b\end{array}$ & $\begin{array}{l}125,43 \pm \\
22,96{ }^{a}\end{array}$ & $\begin{array}{l}12,70 \pm \\
7,00^{a b}\end{array}$ & $\begin{array}{l}111,34 \pm \\
13,55^{\text {a }}\end{array}$ \\
\hline \multirow[t]{8}{*}{$0-10$} & FO & $\begin{array}{l}6,39 \pm \\
0,16^{\mathrm{a}}\end{array}$ & $\begin{array}{l}5,37 \pm \\
0,18^{a}\end{array}$ & $\begin{array}{l}0,63 \pm \\
0,05^{\mathrm{ab}}\end{array}$ & $\begin{array}{l}0,04 \pm 0,00 \\
\mathrm{a}\end{array}$ & $\begin{array}{l}130,20 \pm \\
6,59^{a}\end{array}$ & $\begin{array}{l}2,65 \pm \\
0,21^{\text {a }}\end{array}$ & $\begin{array}{l}102,24 \pm \\
15,54{ }^{a}\end{array}$ \\
\hline & Témoin & $\begin{array}{l}6,42 \pm \\
0,05^{\mathrm{a}}\end{array}$ & $\begin{array}{l}5,28 \pm \\
0,10^{a}\end{array}$ & $\begin{array}{l}0,49 \pm \\
0,10^{\mathrm{a}}\end{array}$ & $\begin{array}{l}0,04 \pm 0,01 \\
\mathrm{a}\end{array}$ & $\begin{array}{l}116,31 \pm \\
11,63^{a}\end{array}$ & $\begin{array}{l}2,83^{ \pm} \\
0,35^{\mathrm{a}}\end{array}$ & $\begin{array}{l}89,22 \pm \\
11,54^{\text {a }}\end{array}$ \\
\hline & Probabilité & 0,0237 & 0,0103 & 0,0000387 & 0,000000803 & 0,0000497 & 0,0052 & 0,000253 \\
\hline & Signification & S & S & HS & THS & THS & HS & HS \\
\hline & $\begin{array}{l}\mathrm{CP}+\text { Enf }+ \\
\mathrm{FO}\end{array}$ & $\begin{array}{l}6,80 \pm \\
0,17^{\mathrm{ab}}\end{array}$ & $\begin{array}{l}5,62 \pm \\
0,26^{\mathrm{ab}}\end{array}$ & $\begin{array}{l}0,85 \pm \\
0,17^{\mathrm{ab}}\end{array}$ & $\begin{array}{l}0,07 \pm 0,01 \\
\mathrm{ac}\end{array}$ & $\begin{array}{l}130,79 \pm \\
10,33 \text { ac }\end{array}$ & $\begin{array}{l}2,18 \pm \\
0,18^{a}\end{array}$ & $\begin{array}{l}146,85 \pm \\
48,92^{a}\end{array}$ \\
\hline & $\mathrm{CP}+\mathrm{FO}$ & $\begin{array}{l}6,99 \pm \\
0,11 \mathrm{ab}\end{array}$ & $\begin{array}{l}5,97 \pm \\
0,15^{\mathrm{ab}}\end{array}$ & $\begin{array}{l}1,01 \pm 0,6 \\
b\end{array}$ & $0,10 \pm 0,01^{c}$ & $\begin{array}{l}257,23 \pm \\
14,34^{\mathrm{C}}\end{array}$ & $\begin{array}{l}8,31 \pm \\
1,43^{a b}\end{array}$ & $\begin{array}{l}155,47 \pm \\
25,31\end{array}$ \\
\hline & CP + Zaï & $\begin{array}{l}7,28 \pm \\
0,21 b\end{array}$ & $\begin{array}{l}6,32 \pm \\
0,23 \mathrm{~b}\end{array}$ & $\begin{array}{l}1,04 \pm 0,6 \\
b\end{array}$ & $\begin{array}{l}0,08 \pm 0,00 \\
\text { bc }\end{array}$ & $\begin{array}{l}169,34 \pm \\
25,27 \text { bc }\end{array}$ & $\begin{array}{l}4,40 \pm \\
0,92^{\mathrm{a}}\end{array}$ & $\begin{array}{l}341,01 \pm \\
62,64 \mathrm{~b}\end{array}$ \\
\hline & Enf + FO & $\begin{array}{l}6,75 \pm \\
0,23^{a}\end{array}$ & $\begin{array}{l}5,86 \pm \\
0,28 \mathrm{ab}\end{array}$ & $\begin{array}{l}0,45 \pm 0,3 \\
\mathrm{a}\end{array}$ & $\begin{array}{l}0,04 \pm 0,00 \\
a b\end{array}$ & $\begin{array}{l}159,31 \pm \\
47,87^{\mathrm{ab}}\end{array}$ & $\begin{array}{l}10,41 \pm \\
5,94 \mathrm{~b}\end{array}$ & $\begin{array}{l}141,33 \pm \\
34,91^{a}\end{array}$ \\
\hline \multirow[t]{4}{*}{$10-20$} & FO & $\begin{array}{l}6,43 \pm \\
0,16^{\mathrm{a}}\end{array}$ & $\begin{array}{l}5,28 \pm \\
0,16^{\mathrm{a}}\end{array}$ & $\begin{array}{l}0,57 \pm 0,6 \\
\mathrm{a}\end{array}$ & $\begin{array}{l}0,04 \pm 0,00 \\
\mathrm{a}\end{array}$ & $\begin{array}{l}128,64 \pm \\
7,31^{\text {a }}\end{array}$ & $\begin{array}{l}1,96 \pm \\
0,28^{a}\end{array}$ & $\begin{array}{l}87,80 \pm \\
16,57^{a}\end{array}$ \\
\hline & Témoin & $\begin{array}{l}6,40 \pm \\
0,08^{a}\end{array}$ & $\begin{array}{l}5,23 \pm \\
0,06^{a}\end{array}$ & $\begin{array}{l}0,50 \pm 0,9 \\
\mathrm{a}\end{array}$ & $\begin{array}{l}0,04 \pm 0,01 \\
\mathrm{a}\end{array}$ & $\begin{array}{l}104,31 \pm \\
14,97^{\text {a }}\end{array}$ & $\begin{array}{l}2,58 \pm \\
0,57^{\mathrm{a}}\end{array}$ & $\begin{array}{l}68,47 \pm \\
5,51^{\text {a }}\end{array}$ \\
\hline & Probabilité & 0,0072 & 0,0046 & 0,000179 & 0,000906 & 0,00074 & 0,0234 & 0,000534 \\
\hline & Signification & HS & HS & THS & THS & THS & S & THS \\
\hline
\end{tabular}


Les valeurs portant les mêmes lettres dans la même colonne ne sont pas statistiquement différentes au seuil de 5 $\%$ (test LSD de Student Newman-Keuls) pour le paramètre chimique considéré. Les chiffres précédés du signe \pm désignent l'erreur standard au sein d'une même pratique agroécologique. N.S : Non Significatif, S : Significatif, H.S : Hautement Significatif et T.S.H : Très Hautement Significatif au seuil de 5 \% (Student Newman-Keuls). * CP : Cordons Pierreux ; Enf : Enfouissement de la paille de céréales ; FO : Fumure organique ; Zaï= il s'agit du zaï avec apport de fumure organique

\section{Effets des pratiques agro-écologiques sur le stock de carbone du sol}

Le Tableau 3 présente la variation de la densité apparente, de la charge en éléments grossiers et du stock de carbone du sol sur la profondeur 0-10 cm sous les différentes pratiques agroécologiques.La pratique CP+ Zaï + FO enregistre la densité apparente la plus élevée $(1,82 \pm 0,11$ $\mathrm{g} / \mathrm{cm}^{3}$ de sol) et la pratique FO la plus faible densité $\left(1,53 \pm 0,04 \mathrm{~g} / \mathrm{cm}^{3} \mathrm{de} \mathrm{sol}\right)$.

La charge en éléments grossiers est plus élevée en CP + FO (35,27 $\pm 6,77 \%)$ et plus faible chez le Témoin (2,65\%). La pratique CP + FO enregistre le stock de carbone le plus élevé $(12,75 \pm 1,06 \mathrm{t} /$ ha), tandis que la pratique Enf + FO présente la plus faible quantité de carbone (6,94 $\pm 1,52 \mathrm{t}$. ha). L'ANOVA a montré une différence significative entre les pratiques AE pour la densité apparente, la charge en éléments grossiers et le stock de carbone (Probabilité < 0,05).

Tableau 3:Variation de la densité apparente, de la charge en éléments grossiers et du stock de carbone sur la profondeur $0-10 \mathrm{~cm}$ au niveau des différentes pratiques agroécologiques

\begin{tabular}{|l|l|l|l|}
\hline Pratiques A-E & D.a (g / $\mathbf{~ c m}^{\mathbf{3}}$ de sol) & Charge en E G ( \%) & Stock C (t / ha) \\
\hline CP+Enf+ FO & $1,57 \pm 0,03^{\mathrm{ab}}$ & $16,21 \pm 3,73^{\mathrm{ac}}$ & $10,45 \pm 0,67^{\mathrm{ab}}$ \\
\hline CP+ FO & $1,81 \pm 0,09^{\mathrm{c}}$ & $35,27 \pm 6,77^{\mathrm{c}}$ & $12,75 \pm 1,06^{\mathrm{b}}$ \\
\hline CP+ Zaï + FO & $1,82 \pm 0,1^{\mathrm{c}}$ & $31,06 \pm 5,55^{\mathrm{bc}}$ & $11,41 \pm 0,89^{\mathrm{ab}}$ \\
\hline Enf+ FO & $1,66 \pm 0,07^{\mathrm{b}}$ & $18,67 \pm 8,01^{\mathrm{ac}}$ & $6,94 \pm 1,52^{\mathrm{a}}$ \\
\hline FO & $1,53 \pm 0,04^{\mathrm{ab}}$ & $6,73 \pm 1,95^{\mathrm{ab}}$ & $9,57 \pm 0,64^{\mathrm{ab}}$ \\
\hline Témoin & $1,55 \pm 0,05^{\mathrm{ab}}$ & $2,65 \pm 1,12^{\mathrm{a}}$ & $8,04 \pm 1,05^{\mathrm{a}}$ \\
\hline Probabilité & 0,0203 & 0,000489 & 0,00681 \\
\hline Signification & $\mathrm{S}$ & THS & HS \\
\hline
\end{tabular}

$\mathrm{CP}=$ Cordons Pierreux $; \mathrm{Enf}=$ Enfouissement $; \mathrm{FO}=$ Fumure Organique $; \mathrm{Zaï}=$ il s'agit du zaï avec apport de fumure organique 
Effets des pratiques agro-écologiques sur les caractéristiques chimiques maje...

\section{Discussion}

L'analyse des données sur les échantillons de sol indique que les pratiques agro-écologiques (AE) intégrant les cordons pierreux améliorent parfois les caractéristiques chimiques du sol par rapport aux pratiques sans cordons pierreux. Cela pourrait s'expliquer par le fait que les cordons pierreux favorisent la sédimentation des particules de matière organique issues des eaux de ruissellement et de la fumure organique. De même, la matière organique est piégée au niveau des trous de zaï, réduisant ainsi les pertes par ruissellement. Plusieurs auteurs $(8,23 ; 24 ; 29)$ ont également montré que la technique du zaï avec apport de fumure organique et combinée ou pas à la réalisation des cordons pierreux amélioraient sensiblement les caractéristiques chimiques du sol.

Les pratiques Zaï + FO et CP +Zaï+ FO ont permis d'augmenter la teneur en matière organique du sol respectivement de 23,19 \% et 17,3 \% par rapport au témoin absolu sur une période de trois ans (28). De même, la pratique du zaï sur une période de six ans a induit des augmentations de $38 \%$ pour le carbone et de $31 \%$ pour l'azote du sol (24). Yaméogo et al.(28) ont montré que la combinaison des cordons pierreux au zaï et le zaï seul augmentaient la teneur en matière organique respectivement de $88,68 \%$ et $113,21 \%$ comparativement au témoin absolu après 10 ans sous forêt en zone sud-soudanienne du Burkina Faso. Ces auteurs précisent également que les teneurs d'azote ont quasiment doublé au cours de la même période pour les mêmes pratiques.

Par ailleurs, Dagnachew et al. (9) en Ethiopie ont rapporté que les techniques CES / DRS à base de terrasses amélioraient les caractéristiques chimiques des sols des champs comparativement aux champs sans terrasses. Toutefois l'apport de la fumure organique et de la paille de céréales en l'absence de dispositifs antiérosifs présente des risques de perte de matière organique par ruissellement et, d'épuisement de nutriments du sol ; ce qui pourrait expliquer les faibles teneurs des caractéristiques chimiques souvent enregistrées. Les cordons pierreux joueraient un rôle dans la lutte contre la dégradation des sols à travers une réduction du ruissellement (30).

Les résultats sur la densité apparente et la charge en éléments grossiers indiquent que les valeurs les plus élevées ont été enregistrées sous techniques CES / DRS. Cela pourrait s'expliquer par la présence d'une forte proportion de graviers et cailloux en raison de la nature gravillonnaire qui caractérisait les sols où étaient mises en œuvre ces techniques. C'est du reste ce qu'ont affirmé les producteurs expérimentateurs. Yaméogo et al. (28) ont obtenu des charges en éléments grossiers du sol de $45 \%$ et $49 \%$ respectivement sur les profondeurs $0-20 \mathrm{~cm}$ et 20-40 cm sous combinaison zaï et cordons pierreux dans la forêt classée de Kuinima en zone sud soudanienne du Burkina Faso. Par contre, Tadesse et al. (25) en Ethiopie ont trouvé que la densité apparente du sol était faible sous techniques CES / DRS comparativement au témoin absolu.

Concernant le stock de carbone, les teneurs sont plus fortes pour les pratiques intégrant les cordons pierreux surtout pour les pratiques $\mathrm{CP}+\mathrm{Zaï}+\mathrm{FO}$ et $\mathrm{CP}+\mathrm{FO}$. Le piégeage de la matière organique et des débris végétaux aussi bien par les cordons qu'au niveau des trous de zaï pourrait expliquer ces valeurs. Nos résultats pourraient aussi s'expliquer par les différences de densité et de charge en éléments grossiers qui sont plus élevées en CP + FO et CP + Zaï + FO. On note en effet que plus la charge en éléments grossiers est importante, plus le stock de carbone est élevé (). Les éléments grossiers par leurs agencements et leurs formes dans le sol offriraient des interstices qui permettent d'accumuler plus facilement le carbone.

Tadesse et al. (25) ont également obtenu des résultats similaires avec les diguettes en terre végétalisées (12,48 t / ha) comparativement au témoin sans dispositif CES / DRS (4,7 t / ha). La 
pratique $\mathrm{CP}+\mathrm{Enf}+\mathrm{FO}$ a présenté une augmentation du stock de 76,22\% par rapport à la pratique Enf + FO ; pour la pratique $\mathrm{CP}+\mathrm{FO}$ cette augmentation est de 34,67\% comparativement à l'apport de la fumure organique (FO). Ces augmentations peuvent être attribuées en grande partie au fait que ces pratiques favorisent la sédimentation de la matière organique provenant des eaux de ruissellement et de la fumure organique, mais également aux différences de densité apparente de sol observées. La combinaison de pratiques intégrant les cordons pierreux semble être la mieux indiquée en termes de stockage de carbone sur la profondeur 0-10 cm du sol. Yaméogo et al. (28) ont enregistré des augmentations du stock de carbone de $166 \%$, $77 \%$ et $21 \%$ respectivement des pratiques de zaï forestier, Zaï forestier combiné aux cordons pierreux et des cordons pierreux comparativement au témoin absolu.

Nos résultats sont également en adéquation avec les travaux de Coulibaly et al. (6), qui ont montré que la pratique de l'agriculture de conservation permettait, au bout de 4 ans, d'accroître le stock de carbone du sol (de 69 \%) comparativement au système conventionnel en zone sud soudanienne du Burkina Faso. Aussi, Mahajan et al. (15) en Inde, rapportait, sur un essai longue-durée, que le stock de carbone du sol était deux à trois fois supérieur sous bourrelets en terre végétalisés que chez le témoin absolu suivant différentes profondeurs du sol.

\section{Conclusion}

La présente étude a été conduite fin d'évaluer à moyen-long terme, les effets des pratiques agro-écologiques sur les caractéristiques chimiques majeures et le stock de carbone du sol (0-10 $\mathrm{cm})$ Les combinaisons de pratiques agro-écologiques intégrant les cordons pierreux ont souvent amélioré les caractéristiques chimiques majeures et le niveau de stock du carbone du sol. Ainsi, des augmentations de stock de carbone de 33,11 \% à 36,67 \% ont été enregistrées au niveau des pratiques comportant les cordons pierreux comparativement à l'apport de la fumure organique seul. Les pratiques intégrant les cordons pierreux et le zaï pourraient contribuer à accroître la productivité des sols de l’Est du Burkina Faso.

\section{Remerciements}

Nos remerciements s'adressent au projet CALAO qui a financé ces travaux. Ils vont également à l'endroit des producteurs qui malgré leurs travaux champêtres sont restés toujours disponibles pour la conduite de l'expérimentation dans leurs champs.

\section{Bibliographie}

1. AFNOR., 1981. Détermination du pH. (Association Française de Normalisation) NF ISO 10390. AFNOR qualité des sols, Paris, 339-348.

2. Aynekulu, E. V., T-G., Shepherd K., Winowiecki L., 2011. A protocol for measurement and Monitoring Soil Carbon Stocks in Agricultural Landscapes. World Agroforestry Centre (ICRAF), $52 \mathrm{p}$.

3. Blanchard M., Coulibaly K., Bognini S., Dugué P. \& Vall E. 2014. Diversité de la qualité des engrais organiques produits par les paysans d'Afrique de l'Ouest : quelles conséquences sur les recommandations de fumure ? Biotechnol. Agron. Soc. Environ 18(4), $12 \mathrm{p}$. 
Effets des pratiques agro-écologiques sur les caractéristiques chimiques maje...

4. Bray R I. I., Kurtz L. T., 1945. Determination of total organic, and available forms of phosphorus in soils. Soil Science, 59: 39-45.

5. Bureau National des Sols (BUNASOLS)., 2000. Etude morpho-pédologique de la province de la Gnagna. Echelle 1 / 100 000. Rapport technique N¹19, 75 p.

6. Coulibaly K., Gomgnimbou A. P. K., Traoré M., Sanon J. F. K., Nacro H. B., 2018. Effets de l'agriculture de conservation sur la dynamique de l'eau et le stock de carbone d'un sol ferrugineux tropical à l'Ouest du Burkina Faso. Sciences et techniques, Sciences naturelles appliquées, numéro spécial, hors-série, 10 p.

7. Coulibaly K., Vall E., Autfray P., Nacro H. B., Sédogo M. P., 2012. Effets de la culture permanente coton-maïs sur l'évolution d'indicateurs de fertilité des sols de l'Ouest du Burkina Faso. Int. J. Biol. Chem. Sci. 6(3): 1069-1080.

8. Dabré A., Hien E., Somé D. Drevon J J., 2017. Effets d'amendements organiques et phosphatés sous zaï sur les propriétés chimiques et biologiques du sol et la qualité de la matière organique en zone soudano-sahélienne du Burkina Faso. Int. J. Biol. Chem. Sci., 11(1): 473-487.

9. Dangnachew M., Moges A., Kebede A., Abebe A., 2020. Effetcs of Soil and Water Conservation Measures on soil quality indicators : the case of Geshy subcathment, Gojeb river Catchment, Ethiopia. Applied and Environmental Soil Science. 16 p.

10. Fontes J., Guinko S., 1995. Carte de la végétation et l'occupation des sols au Burkina Faso. 67 p.

11. Fujisaki K., Chevallier T., Chapuis-Lardy L., Albrecht A., Razafimbelo T., Masse D., Ndour Y B., Chotte J-L, 2017. Soil carbon stock changes in tropical croplands are mainly driven by carbon inputs: A synthesis. Agriculture, Ecosystems and Environment, 259 (2018) :147-158.

12. Hillebrand W. F., Lundel G. E.F., Bright H. A., Hoffman J. I., 1953. Applied inorganic analysis, 2nd ed. John Wiley and Sons, Inc., New York, USA. 1034p.

13. Koulibaly B, Traoré O, Dakuo D, Zombré PN, Bondé D. 2010. Effets de la gestion des résidus de récolte sur les rendements et les bilans culturaux d'une rotation cotonniermaïs-sorgho au Burkina Faso. Tropicultura, 28(3): 184-189.

14. Koulibaly B., Traoré O., Dakuo D., Lalsaga R., Lompo F., Zombré P. N., 2014. Acidification des sols ferrugineux et ferrallitiques dans les systèmes de production cotonnière au Burkina Faso. Int. J. Biol. Chem. Sci. 8(6): 2879-2890.

15. Mahajan G. R., Das B., Manivannan S., Begur L. M., Verma R.R., Desai S., Kulkarni R. M., Latare A. M., Sale R., Murgaonkar D., Patel K. P., Morajkar S., Desai A., Barnes N., Mulla H., 2020. Soil and water conservation measures improve soil carbon sequestration and soil quality under cashews. International Journal of Sediment Research, 64 p.

16. Ministère de l'Agriculture de l'Hydraulique et des Ressources Halieutiques (MAHRH), 2008. Evolution du secteur agricole et des conditions des ménages au Burkina Faso. 
Projet AI/CN-SISA. 92p.

17. Noufé F., Coulibaly K., Kambou A., Traoré S., Tankoano H., 2018. Analyse agroéconomique des technologies de maintien et d'amélioration de la fertilité des sols dans la zone cotonnière de Dano (Burkina Faso). Tropicultura, 36(4): 748-761.

18. Novozansky I. V, J. G. Houba, Vaneck R. and W. Van Vark., 1983. "A novel digestion technic for multi-elernent analysis" Commun Soil Sci. Plant Anal. 239-249.

19. Ouandaogo N., Ouattara B., Pouya B. M., Gnankambary Z., Nacro H. B., Sedogo P. M., 2016 Effets des fumures organo-minérales et des rotations culturales sur la qualité des sols. Int. J. Biol. Chem. Sci. 10(2): 904-918.

20. Ouédraogo M., Dembélé Y., Somé L., 2010. Perceptions et stratégies d'adaptation aux changements des précipitations : cas des paysans du Burkina Faso. Sécheresse, 21 (2) :87-96.

21. Razafimbelo M. T., Andriamananjara A., Rafolisy T., Razakamanarivo H., Masse D., Blanchart E., Falinirina M-V., Bernard L., Ravonjiarison F., Albrech A., 2018. Impact de l'agriculture climato-intelligente sur les stocks de carbone organique du sol à Madagascar. Cah. Agric 35001 (27), 8 p.

22. Rosset M. P., Sosa M. B., Jaime R. M. A., Lozano A. R. D., 2011. The Campesino-toCampesino agroecology movement of ANAP in Cuba: social process methodology in the construction of sustainable peasant agriculture and food sovereignty. The Journal of Peasant Studies, 38 (1): 161-191.

23. Sawadogo H., Bock L., Lacroix D., Zombré N. P., 2008. Restauration des potentialités de sols dégradés à l'aide du zaï et du compost dans le Yatenga (Burkina Faso). Biotechnol. Agron. Soc. Environ. 12(3), 279-290.

24. Somé D., Hien E., Komi Assigbetse, Drevon J. J., Masse D. 2015. Dynamique des compartiments du carbone et de l'azote dans le sol cultivé en niébé et sorgho dans le système zaï en zone Nord soudanienne du Burkina Faso. Int. J. Biol. Chem. Sci. 9(2): 954-969.

25. Tadesse B., Mesfin S., Tesfay G., Abay F., 2016. Effect of integrated soil bunds on key soil properties and soil carbon stock in semi-arid areas of northern Ethiopia. South African Journal of Plant and Soil, $7 \mathrm{p}$

26. Walkley A., Black I. A., 1934. An examination method of the detjareff and a proposed modification of the chromic acid titration method. Soil science. 37. 29-38.

27. World reference base for soil resources (WRB)., 2014. International soil classification system for naming soils and creating legends for soil maps. Food and Agriculture Organization of the United nations, $203 \mathrm{p}$.

28. Yaméogo J. T., Coulibaly K., Compaoré T. M. C., Somé A. N., Nacro H. B., 2019. Contribution of soil and Water Conservation Techniques to soil Carbon Sequestration in a Forest ecosystem in West Africa (Burkina Faso). International Journal of Sciences, 8 (11) : 
Effets des pratiques agro-écologiques sur les caractéristiques chimiques maje...

$32-40$.

29. Yaméogo J. T., Somé A. N., Mette Lykke A., Hien M., Nacro H. B., 2013. Restauration des potentialités de sols dégradés à l'aide du zaï et des cordons pierreux à l'Ouest du Burkina Faso. Tropicultura, 31 (4) : 224-230.

30. Zougmoré R., Ouattara K, Mando A, Ouattara B, 2004. Rôle des nutriments dans le succès des techniques de conservation des eaux et des sols (cordons pierreux, bandes enherbées, zaï et demi-lunes) au Burkina Faso. Science et changements planétaires / Sécheresse, 15 (1), 4-8.

PDF généré automatiquement le 2023-04-26 06:39:51

Url de l'article : https://popups.uliege.be/2295-8010/index.php?id=1862 